\title{
MicroRNA-124-3p inhibits the differentiation of precartilaginous stem cells into nucleus pulposus-like cells via targeting FSTL1
}

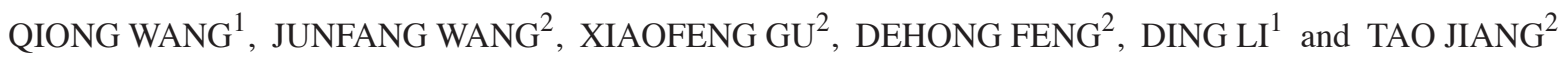 \\ Departments of ${ }^{1}$ Clinical Laboratory and ${ }^{2}$ Orthopedics, Wuxi People's Hospital Affiliated to \\ Nanjing Medical University, Wuxi, Jiangsu 214000, P.R. China
}

Received August 23, 2019; Accepted March 18, 2021

DOI: $10.3892 / \mathrm{etm} .2021 .10157$

\begin{abstract}
MicroRNA (miRNA/miR)-124-3p has been extensively studied in tumor biology and stem cells. However, little is known regarding its functional roles in the differentiation of precartilaginous stem cells (PSCs) into nucleus pulposus-like cells (NPLCs). In the present study, using miRNA microarray screening, it was demonstrated that the miRNA expression profiles differed between rat primary PSCs and TGF- $\beta 1$-induced differentiated NPLCs, and that miR-124-3p was significantly differentially expressed during the differentiation of PSCs to NPLCs. Furthermore, RT-qPCR analysis verified that miR-124-3p expression was decreased during PSC differentiation, with the lowest levels being detected at the later stages. Subsequent experiments revealed that miR-124-3p overexpression significantly decreased the expression of the extracellular matrix proteins, aggrecan and collagen type II, which was accompanied by a significant decrease in follistatin-related protein 1 (FSTL1) expression levels. Moreover, bioinformatics analysis indicated that FSTL1 was a potential target of miR-124-3p, which was additionally verified using luciferase reporter assays. Taken together, these data revealed a specific regulatory pathway of miR-124-3p, which negatively regulated its target gene, FSTL1, during the differentiation of PSCs to NPLCs, and suggested a functional role for miR-124-3p in the differentiation of PSCs.
\end{abstract}

\section{Introduction}

Degeneration of the intervertebral discs is characterized by alterations in the morphology of the discs and the composition of the extracellular matrix, including a reduction in the number of the intervertebral disc cells $(1,2)$. It has been reported that when the intervertebral disc degenerates, the extracellular

Correspondence to: Dr Junfang Wang, Department of Orthopedics, Wuxi People's Hospital Affiliated to Nanjing Medical University, 299 Qingyang Road, Wuxi, Jiangsu 214000, P.R. China

E-mail: wjf801109@163.com

Key words: microRNA-124-3p, precartilaginous stem cells, nucleus pulposus-like cells matrix is reduced, resulting in a decrease in the water content of the intervertebral disc, thereby resulting in the degeneration of the intervertebral disc structure (1-4).

Tissue engineering has been increasingly employed in the medical field as a treatment for degenerative discs, with cell therapy becoming a potential treatment for intervertebral disc degeneration (5). Previous studies have reported that various types of cells, including nucleus pulposus cells, chondrocytes and mesenchymal stem cells (MSCs), can be used for cell therapy (6-8). With further progress, stem cell therapy has been applied in the clinic, with several studies reporting these results. For example, Centeno et al (9) reported that patients with degenerative disc disease (DDD) who are treated with MSCs to counteract lower back pain with radicular symptoms, exhibit minor adverse effects and considerable improvements in the degree of pain, function and overall quality of life during a 6 year follow-up. Li et al (10) investigated the characteristics of stem cells, including nucleus pulposus-derived stem cells (NPSCs), annulus fibrosus-derived stem cells (AFSCs) and cartilage endplate-derived stem cells (CESCs), in human degenerative intervertebral discs to determine the best stem cell-like characteristics. No significant differences in cell morphology among NPSCs, AFSCs and CESCs have been revealed; however AFSCs have been indicated to exhibit the best stem cell-like characteristics in the human degenerative intervertebral disc (10).

The present study focused on another type of stem cell, namely precartilaginous stem cells (PSCs). It has been indicated that PSCs are localized in the perichondrium surrounding the epiphysis (11). PSCs are adult stem cells with a multi-directional differentiation potential, which have been reported to differentiate into chondrocytes and osteoblasts to promote the growth of animal limbs (12). PSCs have been isolated and purified via immunomagnetic bead sorting, and used as seed cells in several tissue engineering studies (12). The discovery of PSCs has provided a novel tool for cell transplantation to repair degenerative intervertebral discs (13). PSCs have been indicated to serve an important role in cartilage growth and internalization, as well as articular cartilage injury repair (14). PSCs are precursor cells of chondrocytes and exhibit a homology with intervertebral disc nucleus cells. The alterations in nucleus pulposus cells have been reported to serve an important role in the degeneration of intervertebral discs (15). The nucleus pulposus is an avascular 
tissue with a limited number of cells and it is difficult to repair its structure following the occurrence of a lesion (16). Therefore, various studies have investigated the use of seed cells to repair degenerated nucleus pulposus cells (17-19). The nucleus pulposus cells and chondrocytes of the intervertebral disc have been reported to exhibit numerous common characteristics, such as production of collagen II and aggrecan as principal components of the extracellular matrix $(18,19)$. Moreover, nucleus pulposus-like cells (NPLCs), which are derived from the degenerated intervertebral disc, have been indicated to undergo osteogenesis and promote cartilage repair owing to the fact that these cells secrete factors (TGF-1, IL-1, TNF, prostaglandin E2, IL-10, and granulocyte-macrophage colony stimulating factor) which promote the proliferation and regulate the differentiation of chondrocytes, as well as promote the synthesis of extracellular matrix components (20).

Our previous study indicated that PSCs can be induced to differentiate to NPLCs to repair degenerative intervertebral discs with unique therapeutic advantages (21). However, the detailed mechanisms of action behind the differentiation are still unclear. MicroRNAs (miRNAs/miRs) are a class of small noncoding RNA molecules that negatively regulate gene expression at the post-transcriptional level (22). It has been reported that miRNAs not only regulate several normal physiological processes but are also associated with the development of DDD (23). In the present study, miRNA microarray screening was employed to identify differences in miRNA expression profiles between TGF- $\beta 1$-induced rat primary PSCs and differentiated NPLCs. miR-124-3p was verified as a significantly differentially expressed miRNA during the differentiation of PSCs to NPLCs. Moreover, the mechanisms of action through which miR-124-3p regulates the fate of PSCs and its role in the differentiation process were also investigated.

\section{Materials and methods}

Chemicals, reagents and antibodies. TGF- $\beta 1$ was purchased from Selleck Chemicals. miR-124-3p and negative control (NC/nc) agomirs (cat. no. miR40000422-4-5) were obtained from Guangzhou RiboBio Co., Ltd. Primary antibodies against fibroblast growth factor receptor 3 (FGFR-3; cat. no. ab133644), follistatin-related protein 1 (FSTL1; cat. no. ab232777,), collagen II (cat. no. ab188570), aggrecan (cat. no. ab3778) and HRP-conjugated secondary antibody GAPDH (cat. no. ab181602) were obtained from Abcam. FSTL1 small interfering (si)RNA and siRNAnc sequences were designed, synthesized and validated by Shanghai GenePharma Co., Ltd. The siRNA sequences used were as follows: FSTL1, 5'-GCAAAUACUUACGGACUU UU-3'; and siRNAnc, 5'-GCAUCUUGAGAUUUAAUCA-3'. FSTL1 (cat. no. HP100806), TPST2 (cat. no. HP101143) and enhancer of zeste homolog 2 (EZH2; cat. no. HP101222) quantitative PCR (qPCR) primers were purchased from Sino Biological Inc. (Shanghai, China). COS7 and 293 cells were purchased from the Cell Bank of the Chinese Academy of Sciences.

Primary PSCs culture. PSCs were generated as previously described (11). Briefly, the perichondrial mesenchyme (the rings of LaCroix) were isolated from Sprague Dawley rats (Trophic Animal Feed High-Tech Co., Ltd.) and subsequently digested in $0.25 \%$ Trypsin Solution (cat. no. 25200072; Thermo Fisher Scientific, Inc.) supplemented with $0.05 \%$ collagenase type I (Sigma-Aldrich; Merck KGaA) for 3-5 min at $37^{\circ} \mathrm{C}$. The digestion mixture was resuspended in FBS. Subsequently, the cells were dispersed and resuspended as a single cell suspension in 0.1 M PBS with centrifugation speeds of $167.7 \mathrm{x} g$ for $5 \mathrm{~min}$ at room temperature. The cell suspension was incubated with the FGFR-3 antibody (1:500; cat. no. ab133644; Abcam) for $15 \mathrm{~min}$ at $4^{\circ} \mathrm{C}$, and subsequently, an immunomagnetic separation system (Miltenyi Biotec $\mathrm{GmbH}$ ) was used to purify the FGFR-3 expressing cells. The isolated PSCs were cultured in complete DMEM/F12 medium supplemented with $20 \%$ FBS (Nanjing KeyGen Biotech Co., Ltd.) at $37^{\circ} \mathrm{C}$ with $5 \% \mathrm{CO}_{2}$. The phenotype identification of the rat PSCs was based on the FGFR-3 protein expression, which was detected via western blotting. The present study was approved by the Institutional Animal Care and Use Committee of Wuxi People's Hospital (approval no. WXPH20190103c0600105). In the current study, 60 rats were used between January and March 2019. Animal health and behavior were monitored daily. All operations were performed according to international guidelines for the care and treatment of experimental animals. The rats were male Sprague-Dawley rats of 5-7 weeks of age weighing 150-200 g. Cages were cleaned and enrichment items were renewed weekly. The animal room had a controlled 12-h light/dark cycle (lights on at 6:00 $\mathrm{AM})$, temperature $\left(22 \pm 2^{\circ} \mathrm{C}\right)$ and relative humidity (45-65\%). Daily food and water were supplied by laboratory staff. The rats were maintained in an ordinary housing facility, which is in accordance with the national standard 'Laboratory Animal-Requirements of Environment and Housing Facilities' (standard no. GB 14925-2010). The animals were euthanized in accordance with the requirements of the 'Guidelines for the Examination of the Scientific Research of Experimental Animal Welfare' (standard no. GB/T 35892-2018) using intravenous administration of pentobarbital $(100-150 \mathrm{mg} / \mathrm{kg})$ and death was verified via examining the animals' heartbeat for complete cessation and pupil dilation.

PSCs cell differentiation. PSCs were differentiated according to a previously published protocol (24). Briefly, PSCs at passage three were trypsinized and transferred to six-well plates at a concentration of $1 \times 10^{5}$ cells $/ \mathrm{ml}$. Differentiation of PSCs was induced by culturing the cells in DMEM/F12 medium supplemented with $10 \% \mathrm{FBS}, 10 \mathrm{ng} / \mathrm{ml}$ TGF- $\beta 1,100 \mathrm{nM}$ dexamethasone, $50 \mu \mathrm{g} / \mathrm{ml} \mathrm{L}$-ascorbic acid 2-phosphate, $100 \mu \mathrm{g} / \mathrm{ml}$ sodium pyruvate, $40 \mu \mathrm{g} / \mathrm{ml}$ proline and $6.25 \mathrm{mg} / \mathrm{l}$ insulin at $37^{\circ} \mathrm{C}$ with $5 \% \mathrm{CO}_{2}$ for 7 days.

Cell lines and culturing conditions. COS-7 and 293 cells were cultured in DMEM (Gibco; Thermo Fisher Scientific, Inc.) supplemented with $10 \%$ fetal bovine serum (FBS; Gibco; Thermo Fisher Scientific, Inc.) and maintained in an incubator at $37^{\circ} \mathrm{C}$ and $5 \% \mathrm{CO}_{2}$. The medium was replaced 2-3 times a week. When the cell density reached $70-80 \%$, cells were digested by $0.25 \%$ trypsin (Gibco; Thermo Fisher Scientific, Inc.) and passaged.

Cell transfection. Small interfering RNA targeting FSTL1 (siFSTL1) was synthesized by Guangzhou RiboBio Co., Ltd., and a scrambled siRNA (siNC; Guangzhou RiboBio Co., Ltd.) 
was used as the negative control. One day before transfection, cells were digested with $0.25 \%$ trypsin (cat. no. C0202; Beyotime Institute of Biotechnology). Then, $50 \mu 1$ of Opti-MEM $^{\circledR}$ medium (cat. no. 31985062; Invitrogen; Thermo Fisher Scientific, Inc.) was mixed with siFSTL1(cat. $n$ o. siB14715103107-1-5; Guangzhou RiboBio Co., Ltd.) or siNC. And $3 \mu$ l Lipofectamine ${ }^{\circledR} 3000$ reagent (cat. no. L3000015; Thermo Fisher Scientific, Inc.) was diluted with $50 \mu \mathrm{l}$ OptiMEM. Then, the above two mixtures were mixed. Then cells were transfected with the mixture according to the Lipofectamine $^{\circledR} 3000$ kit (Thermo Fisher Scientific, Inc.).

miR-124-3p agomir (miR40000422-4-5 Guangzhou RiboBio Co., Ltd.) and negative control (agomir NC) were synthesized by Guangzhou RiboBio Co., Ltd. Cells were seeded in six-well plates $\left(1 \times 10^{5}\right.$ cells $\left./ \mathrm{ml}\right)$ and transfected with $50 \mathrm{nM}$ miR-124-3p agomir or $50 \mathrm{nM}$ agomir NC using Lipofectamine $^{\circledR} 3000$ kit (Thermo Fisher Scientific, Inc.). The transfection efficiency was observed by a fluorescence microscope after $24 \mathrm{~h}$.

miRNA microarray analysis. Total RNA from rat primary PSCs and TGF- $\beta 1$-differentiated NPLCs was isolated using the TRIzol ${ }^{\circledR}$ reagent (Invitrogen; Thermo Fisher Scientific, Inc.) according to the manufacturer's instructions. Following quantification of total RNA using NanoDrop ND-1000 Ultraviolet Spectrophotometer (NanoDrop Technologies; Thermo Fisher Scientific, Inc.), a miRNA profiling system (Agilent Technologies, Inc.) was used to detect the differences in miRNA expression between primary PSCs and NPLCs. The slides were scanned using a microarray scanner (GenePix 4100A) and the data were quantified using Feature Extraction software v10.7 (both from Agilent Technologies, Inc.). The GeneSpring GX software v12.6 (Agilent Technologies, Inc.) was additionally used to analyze the miRNA expression data that were detected using the microarray system.

Reverse transcription-quantitative $(R T-q) P C R$. Total RNA of PSCs was extracted using TRIzol ${ }^{\circledR}$ reagent (Invitrogen; Thermo Fisher Scientific, Inc.). The primers used for the amplification of miR-124-3p (cat. no. miRA1001726-1-100) and U6 endogenous control (cat. no. miRAN0002-1-100) were purchased from Guangzhou RiboBio Co., Ltd. Subsequently, qPCR was performed using the Mir-X ${ }^{\mathrm{TM}}$ miRNA RT-qPCR TB Green ${ }^{\circledR}$ kit according to the manufacturer's instructions (Takara Biotechnology). Briefly, Total RNA was reverse transcribed to obtain cDNA. The reverse transcription process of miR-124-3p used the Mir-X miRNA First-Strand Synthesis Kit (cat. no. 638313; Takara Biotechnology, Co., Ltd.), the tube was incubated for $1 \mathrm{~h}$ at $37^{\circ} \mathrm{C}$, and the reaction terminated at $85^{\circ} \mathrm{C}$ for $5 \mathrm{~min}$ to inactivate the enzymes. The PCR reactions were carried out using TB Green Advantage qPCR Premix (cat. no. 639676; Takara Biotechnology, Co., Ltd.) under the following conditions: Denaturation, $95^{\circ} \mathrm{C} 10 \mathrm{sec}$; qPCR x 40 cycles of $95^{\circ} \mathrm{C} 5 \mathrm{sec}$ and $60^{\circ} \mathrm{C} 20 \mathrm{sec}$; dissociation curve, $95^{\circ} \mathrm{C} 60 \mathrm{sec}, 55^{\circ} \mathrm{C} 30 \mathrm{sec}$ and $95^{\circ} \mathrm{C} 30 \mathrm{sec}$. The relative miRNA expression was calculated using the $2^{-\Delta \Delta \mathrm{Cq}}$ method (25).

Target gene prediction. To predict the potential target genes of miRNA-124-3p,three bioinformatics analysis tools, TargetScan v7.2 (www.targetscan.org),Pictar(https://pictar.mdc-berlin.de/) and miRanda (http://www.microrna.org/microrna/home.do) were used to predict its potential target genes.

Luciferase reporter assay. For the luciferase reporter assay, a construct containing the 3'-untranslated region (UTR) of the FSTL1 mRNA carrying the putative miRNA-124-3p binding site or a respective mutant, which was used as a control, were cloned into the pGL3 Luciferase Reporter plasmid (Promega Corporation). Cells $\left(1 \times 10^{5}\right)$ were co-transfected with a $50 \mathrm{ng} /$ well reporter plasmid and $50 \mathrm{nM}$ miR-124-3p agomir or $50 \mathrm{nM}$ agomir NC in a 6-well plate for $48 \mathrm{~h}$. Firefly and Renilla luciferase activities were detected using Dual-Luciferase ${ }^{\circledR}$ Reporter Assay System (cat. no. E1910; Promega Corporation). Briefly, $500 \mu \mathrm{l}$ of PLB was added and agitated for $15 \mathrm{~min}$. LAR II $(100 \mu \mathrm{l})$ and cells $(20 \mu \mathrm{l})$ were sequentially added to 96-well plate. Then, $100 \mu 1$ of $1 \mathrm{X}$ Stop \& Glo reagent was added to detect the luminescence intensity of Renilla luciferase.

Western blot analysis. Following miR-124-3p agomir or FTSL1 siRNA transfection, PSCs were collected and solubilized in RIPA lysis buffer (cat. no. KGP702; Nanjing KeyGen Biotech Co., Ltd.). Following centrifugation for $10 \mathrm{~min}$ $\left(1,600 \times \mathrm{g}, 4^{\circ} \mathrm{C}\right)$, the protein concentration was measured using a BCA assay kit (Sigma-Aldrich; Merck KGaA). The samples (20 $\mu \mathrm{g}$ /well) were separated on 10\% SDS-PAGE (Nanjing Genscript Biotechnology Co., Ltd.) at $140 \mathrm{~V}$ for $50 \mathrm{~min}$. Subsequently, the separated proteins were transferred onto PVDF membranes at $300 \mathrm{~mA}$ for $60 \mathrm{~min}$ (Nanjing Genscript Biotechnology Co., Ltd.). The membranes were subsequently blocked for $1 \mathrm{~h}$ at room temperature with $5 \%$ fat-free dried milk diluted in TBS-0.1\% Tween-20, followed by incubation with primary antibodies against FSTL1 $(1: 1,000)$, collagen II $(1: 1,000)$, with GAPDH being used as an internal control (1:80,000), at $4^{\circ} \mathrm{C}$ overnight. The membranes were subsequently incubated with HRP-conjugated secondary antibodies (anti-rabbit; cat. no. ab6721; 1:10,000; Abcam) for $1 \mathrm{~h}$ at room temperature. The protein bands were detected using an ECL chemiluminescence kit (Nanjing KeyGen Biotech Co., Ltd., China) and semi-quantified using ImageQuant TL analysis software v8.1 (Cytiva).

Statistical analysis. All statistical analyses were performed using SPSS v19 software (IBM Corp.). Each experiment was repeated three times and data were presented as the mean \pm SD. Unpaired Student's t-test or one-way ANOVA followed by Bonferroni's post hoc test were applied to identify the statistical significance. $\mathrm{P}<0.05$ was considered to indicate a statistically significant difference.

\section{Results}

miR-124-3p is associated with the differentiation of PSCs to NPLCs. To identify miRNAs that are associated with the regulation of the differentiation of PSCs to NPLCs, miRNA microarray technology was used to detect differences in miRNA expression profiles between TGF- $\beta 1$-treated rat primary PSCs (day 0) and differentiated NPLCs (day 15). The efficient induction of PSC differentiation by TGF- $\beta 1$ has been previously verified in our previous study (16). The results of the present study indicated that when compared 

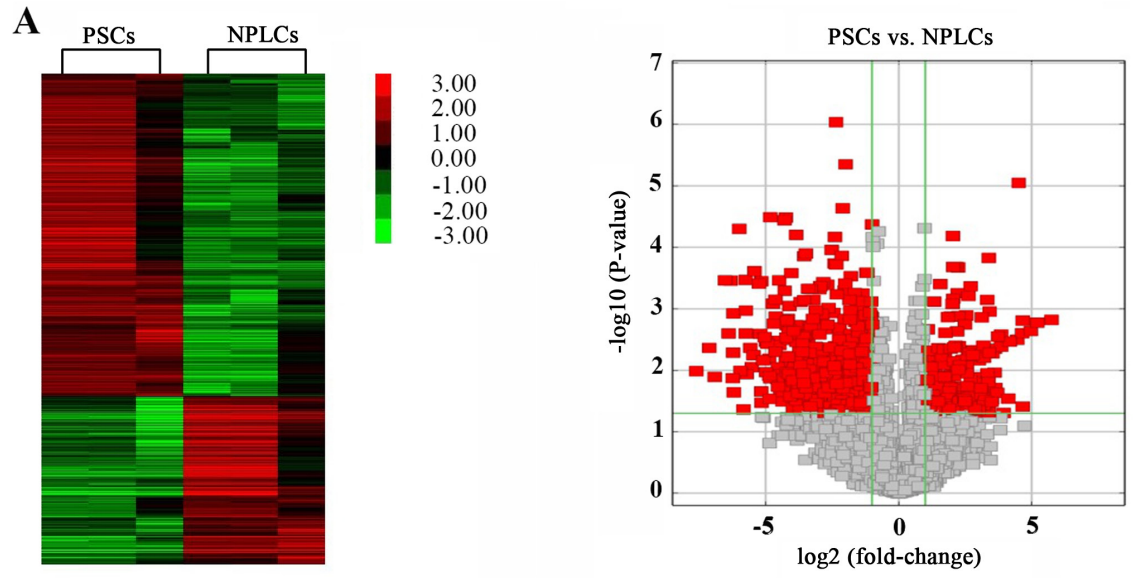

B
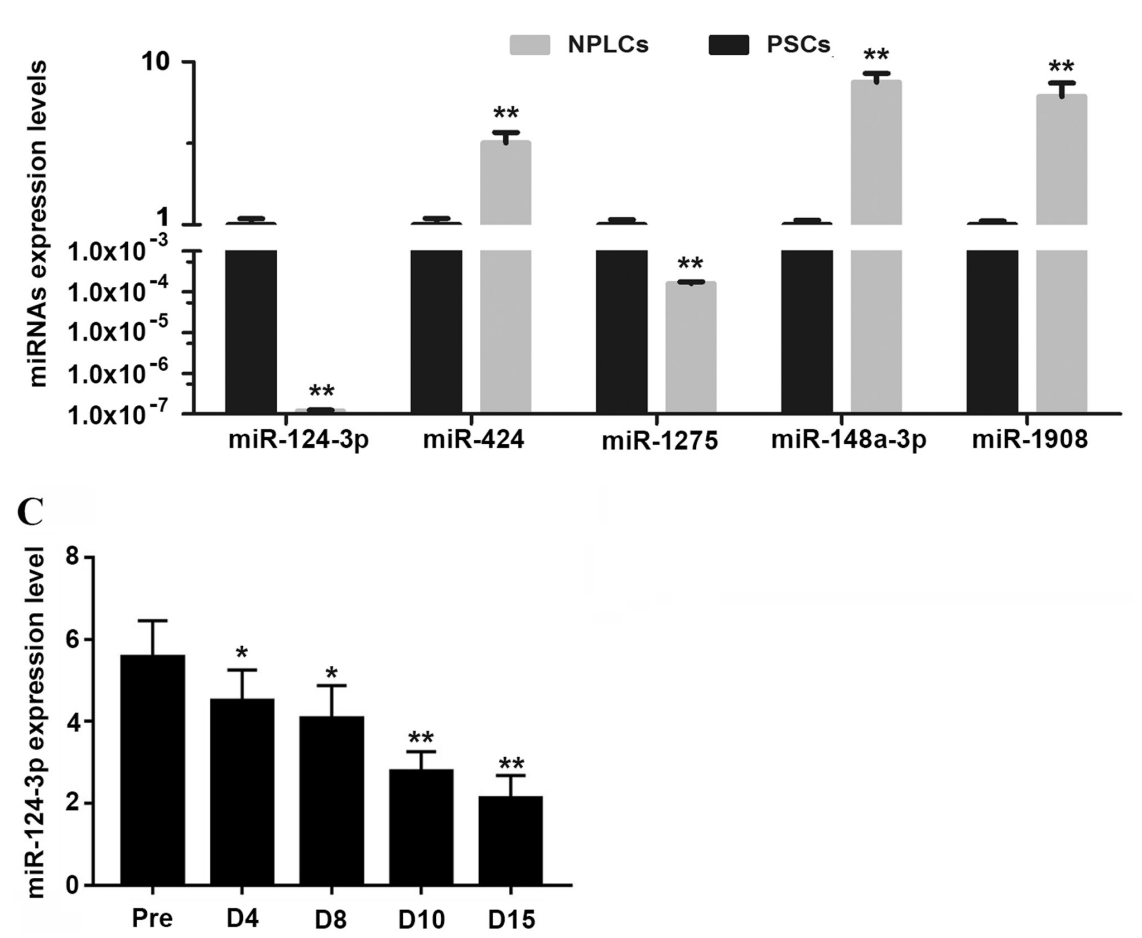

Figure 1. miR-124-3p participates in the differentiation of PSCs to NPLCs. (A) The differences in miRNA expression profiles were detected using a miRNA microarray. (B) The indicated miRNAs were validated using RT-qPCR. ${ }^{* *} \mathrm{P}<0.01$ vs. PSCs. (C) The expression of miR-124-3p during the differentiation of PSCs was detected using RT-qPCR. "P<0.05 and ${ }^{* *} \mathrm{P}<0.01$ vs. Pre (D0) group. miRNA/miR, microRNA; PSCs, precartilaginous stem cells; NPLCs, nucleus pulposus-like cells; RT-qPCR, reverse transcription-quantitative PCR; D, day.

with primary PSCs, 50 miRNAs in the differentiated NPLCs were considerably upregulated ( $>3$-fold), while 36 miRNAs were downregulated $<3$-fold (Fig. 1A). A total of five miRNAs (miR-124-3p, miR-424, miR-1275, miR-148a-3p and miR-1908) with a $>5$-fold expression difference and a small within group variation were selected for subsequent validation using RT-qPCR. As indicated in Fig. 1B, the expression tendency of the five miRNAs, was consistent with the results of the microarray screening. Among them, the difference in the expression levels of miR-124-3p was the lowest (Fig. 1B).

The alterations in miR-124-3p expression during the differentiation of PSCs to NPLCs were subsequently analyzed (between days 0 to 15). PSCs were induced to differentiate into NPLCs via treatment with TGF- $\beta 1$ and the expression levels of miR-124-3p at various time points of differentiation were detected using RT-qPCR (days 0, 4, 8, 10 and 15). The results verified that the expression of miR-124-3p decreased along with the differentiation of PSCs and exhibited the lowest levels during the later stages of differentiation (Fig. 1C).

miR-124-3p inhibits the differentiation of PSCs. As specific markers for nucleus pulposus cells have not been identified, collagen II and aggrecan are used to detect differentiated nucleus pulposus cells (26). Therefore, the expression of collagen II and aggrecan during the differentiation of PSCs into NPLCs was detected using western blotting. It was demonstrated that collagen II and aggrecan protein were expressed at a significantly higher levels in NPLCs compared with PSCs, which suggested that PSCs were efficiently differentiated into NPLCs (Fig. 2A). Furthermore, to identify 


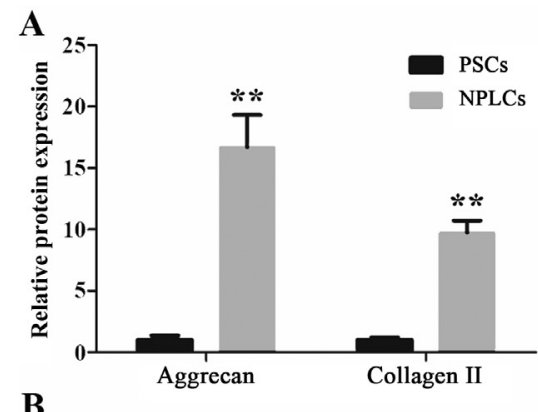

B

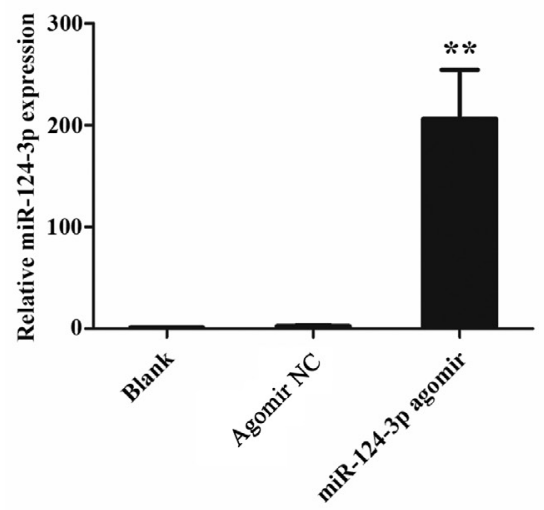

C

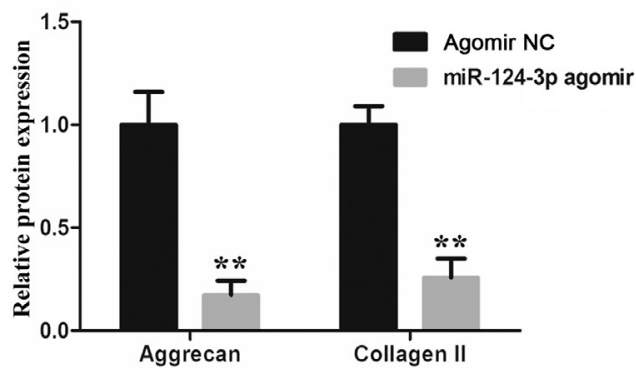

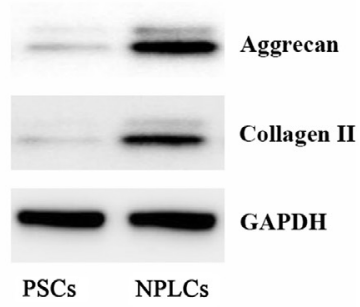

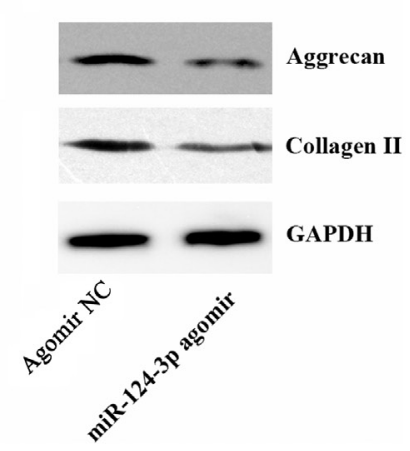

Figure 2. Effect of miR-124-3p in the differentiation of PSCs to NPLCs. (A) The expression of collagen II and aggrecan in PSCs and NPLCs was analyzed via western blotting. ${ }^{* *} \mathrm{P}<0.01$ vs. PSCs. (B) The expression of miR-124-3p in PSCs transfected with miR-124-3p agomir was detected via reverse transcription-quantitative PCR. ${ }^{* *} \mathrm{P}<0.01$ vs. agomir NC or Blank. (C) The expression of collagen II and aggrecan following transfection with miR-124-3p agomir was examined via western blotting. ${ }^{* *} \mathrm{P}<0.01$ vs. agomir NC. miR, microRNA; PSCs, precartilaginous stem cells; NPLCs, nucleus pulposus-like cells; NC, negative control.

the role of miR-124-3p in PSCs differentiation, miR-124-3p was overexpressed in PSCs via transfection with miR-124-3p agomir (Fig. 2B). As presented in Fig. 2C, collagen II and aggrecan protein expression levels in the miR-124-3p agomir group were significantly decreased when compared with the NC group on day 18 post-transfection. These results suggested that miR-124-3p overexpression inhibited the differentiation of PSCs into NPLCs.

miR-124-3p targets FSTL1 during PSCs differentiation. Using TargetScan software, 7-9 binding sites were predicted in the 3'-UTR region of the FSTL1 mRNA. The results indicated that FSTL1, EZH2 and TPST2 may represent potential target genes of miR-124-3p (Fig. 3A). The alterations in the expression levels of the aforementioned three genes during the differentiation process of PSCs (0, 4, 8 and 15 days) were subsequently determined. As indicated in Fig. 3B, only FSTL1 was demonstrated to be gradually increased during the differentiation process, in contrast to the expression levels of miR-124-3p, which were indicated to decrease during PSCs differentiation, as aforementioned. However, the expression of EZH2 and TPST2 showed neither gradual increase nor decrease. Notably, the majority of miRNAs have been indicated not to affect the mRNA expression of their target gene. However, certain miRNAs have been reported to result in mRNA degradation of their target genes (27). In the present study, it was demonstrated that miR-124-3p overexpression resulted in decreased FSTL1 mRNA levels in PSCs (Fig. 3C).

Moreover, in order to verify whether miR-124-3p can directly bind to and regulate the mRNA expression levels of FSTL1, a dual-luciferase reporter assay was used in the present study. Luciferase reporter plasmids containing the predicted GUGCCUU binding site of the 3'-UTR of FSTL1 and a mutant site (UGCUUGC) were constructed and co-transfected with miR-124-3p into COS7 and 293 cells to detect the alterations in luciferase activity. As indicated in Fig. 4A, the results 
A

Position 402-409 of TPST2 3' UTR
rno-miR-124-3p
Position 33-39 of EZH2 3' UTR
rno-miR-124-3p
Position 1517-1523 of FSTL1 3' UTR
rno-miR-124-3p

B

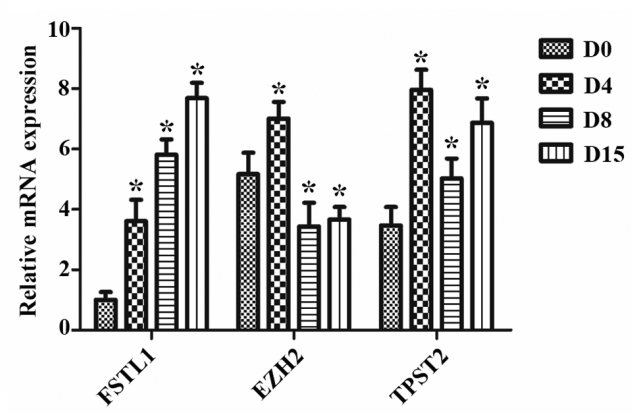

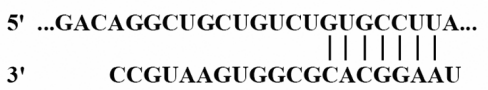

$5^{\prime}$...CUCCUCUCAAACAGCUGCCUUAG...

3' CCGUAAGUGGCGC-ACGGAAU
5 '...CAACUCCCUCUCCAAGUGCCUUG...
3' CCGUAAGUGGCGCACGGAAU

C

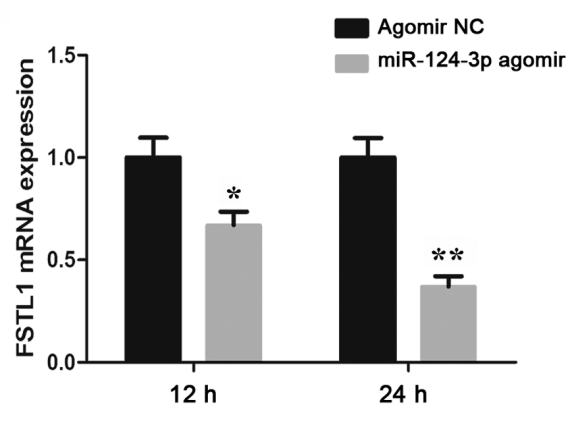

Figure 3. Analysis of the targets of miR-124-3p during PSCs differentiation. (A) Bioinformatics analysis tools predicted the potential target genes for miR-124-3p. (B) The expression of FSTL1, EZH2 and TPST2 during the differentiation of PSCs to nucleus pulposus-like cells was detected via RT-qPCR. "P<0.05 vs. D0. (C) PSCs were transfected with miR-124-3p agomir, followed by the detection of FSTL1 mRNA levels via RT-qPCR. "P<0.05 and " $\mathrm{P}<0.01$ vs. agomir NC. miR, microRNA; PSCs, precartilaginous stem cells; RT-qPCR, reverse transcription-quantitative PCR; D, day; UTR, untranslated region; FSTL1, follistatin-related protein 1; TPST2, protein-tyrosine sulfotransferase 2; EZH2, enhancer of zeste homolog 2; NC, negative control.

revealed that miR-124-3p overexpression significantly reduced the luciferase activity of FSTL1 in the wild-type group when compared with the mutant group. Furthermore, western blot analysis revealed that miR-124-3p overexpression resulted in a decrease in FSTL1 expression in both PSCs (day 0) and NPLCs (day 15) compared with the control group (Fig. 4B). Subsequently, FSTL1 was knocked down using an FSTL1 siRNA (Fig. 4C) and the expression of collagen II and aggrecan was determined. The results revealed that FSTL1 knockdown resulted in decreased collagen II and aggrecan protein expression levels compared with control cells, which indicated that FSTL1 was associated with the differentiation of PSCs (Fig. 4D).

\section{Discussion}

PSC-derived NPLCs may be used to treat intervertebral discs degeneration, owing to the capacity of NPLCs to promote tissue remodeling, which has been shown to result in lower back pain relief (28). The present study demonstrated that miR-124-3p was associated with the differentiation of PSCs into NPLCs and that the expression of miR-124-3p gradually decreased during the differentiation process, from day 0 to day 15 . Previous studies have indicated that miR-124-3p expression is decreased in Alzheimer's disease (AD) and several types of tumors, including bladder cancer, hepatocellular carcinoma and glioma (29-32). Recently, Zhang et al (33) reported that miR-124-3p attenuates neuropathic pain induced by chronic sciatic nerve injury in rats. However, the function of miR-124-3p in lower back pain, which is induced by DDD, remains unknown. Therefore, the present study aimed to elucidate the molecular mechanism of action underlying the association between miR-124-3p and the differentiation of PSCs into NPLCs, and to identify the potential target genes which may be involved in this process.

In AD, Kang et al (29) demonstrated that upregulation of miR-124-3p expression levels attenuates cell apoptosis and abnormal hyperphosphorylation of Tau protein in N2a/APP695swe cells. Zo and Long (30) reported that inhibition of miR-124-3p expression increases bladder cancer cell proliferation, migration and invasion, as well as reducing apoptosis. Furthermore, Luo et al (32) revealed that transfection with miR-124-3p inhibits the expression of cell cycle and epithelial-mesenchymal transition regulators, and inhibits the proliferation, migration and invasion of glioma cells.

To explore the function of miR-124-3p in the differentiation of PSCs to NPLCs, miR-124-3p was overexpressed in PSCs using a miR-124-3p agomir. Collagen type II and aggrecan have been indicated to be produced by nucleus pulposus cells and are required for their homeostasis $(34,35)$. The results of the present study revealed that collagen II and aggrecan levels were increased during the differentiation of PSCs to NPLCs. However, following transfection of PSCs with miR-124-3p agomir, the expression of collagen II and aggrecan in NPLCs (day 18) was significantly decreased when compared with the control group. This suggested that miR-124-3p may influence the differentiation of PSCs into NPLCs.

However, the mechanism of action through which miR-124-3p regulates the differentiation of PSCs remained unclear. Bioinformatics analysis indicated that FSTL1, EZH2 and TPST2 may represent targets of miR-124-3p during the differentiation of PSCs. However, the results indicated that 
A

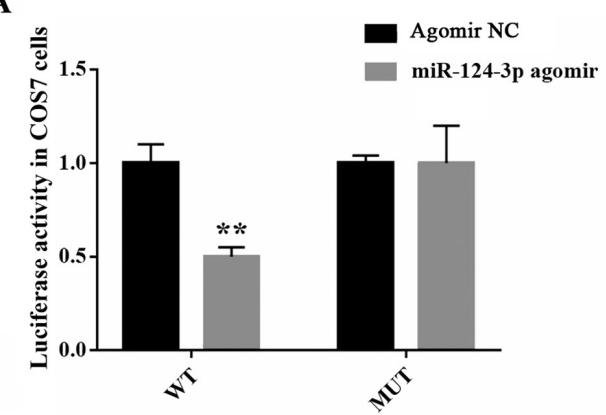

B

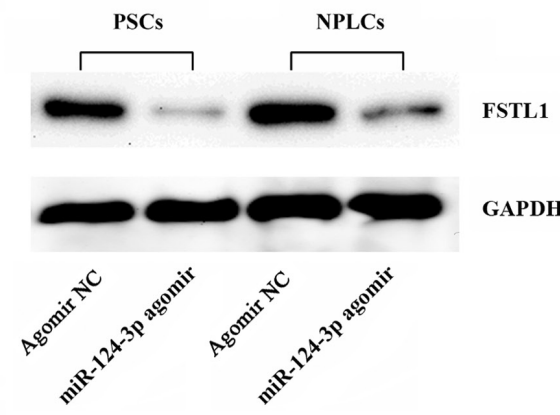

C

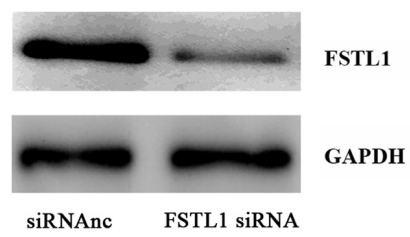

D

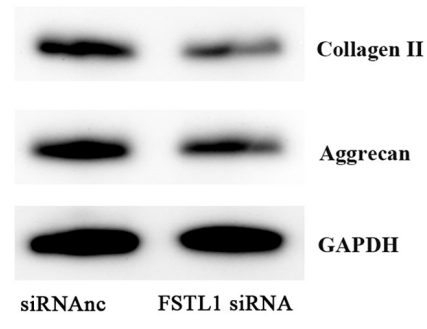

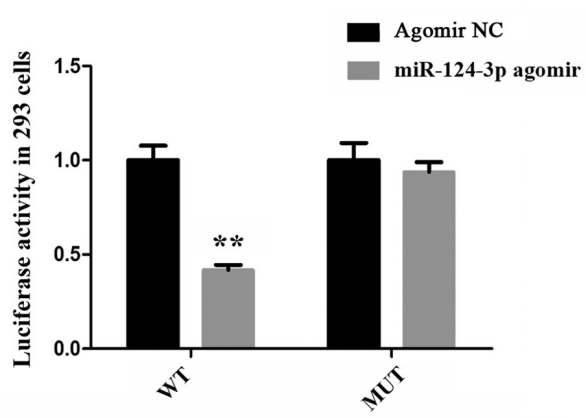
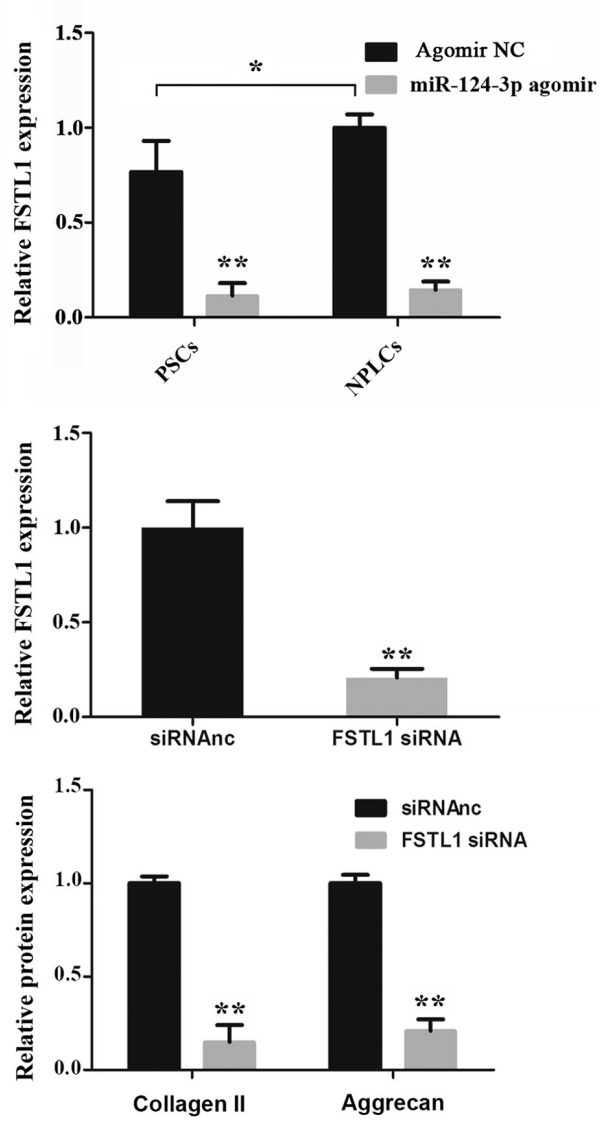

Figure 4. miR-124-3p directly regulates FSTL1 function. (A) Luciferase reporter plasmids containing the predicted GUGCCUU binding site of the 3'-untranslated region of FSTL1 and its mutant homolog (UGCUUGC) were constructed and co-transfected with miR-124-3p into COS7 and 293 cells to detect the alterations in luciferase activity. " P $<0.01$ vs. agomir NC. (B) The effects of miR-124-3p overexpression on FSTL1 expression during the differentiation of PSCs to NPLCs was analyzed via western blotting. " $\mathrm{P}<0.05$ vs. PSCs; ${ }^{* *} \mathrm{P}<0.01$ vs. agomir NC. (C) The efficiency of FSTL1 siRNA was verified via western blotting. ${ }^{* *} \mathrm{P}<0.01$ vs. siRNAnc. (D) The expression of collagen II and aggrecan following FSTL1 knockdown was detected via western blotting. "P<0.01 vs. siRNAnc. miR, microRNA; MUT, mutant; PSCs, precartilaginous stem cells; NPLCs, nucleus pulposus-like cells; NC/nc, negative control; FSTL1, follistatin-related protein 1; si, small interfering; WT, wild-type.

only FSTL1 levels increased gradually during the differentiation of PSCs to NPLCs. Considering that miRNAs recognize their target genes via complementary base pairing and subsequently guide the silencing complex to degrade or repress the translation of the target mRNA according to the degree of complementarity (27), it was speculated that FSTL1 may be a potential target gene of miR-124-3p. FSTL1 was firstly identified in mouse osteoblasts and as TGF- $\beta 1$ has been indicated to induce its upregulation, FSTL1 is also known as TGF- $\beta$-stimulated clone-36 (36). FSTL1 is an extracellular matrix protein, which is widely expressed in all eukaryotic cells and has been associated with cell differentiation, metabolism, cell proliferation and the immune response (37-39). A previous study indicated that FSTL1 also participates in the regulation of Lumbar disc herniation (40). It was demonstrated that FSTL1 expression is increased during the progression of intervertebral disc disease and promotes inflammatory reactions in the nucleus pulposus via the MAPK and NF- $\kappa \mathrm{B}$ signaling pathways (40). The present study revealed that FSTL1 was also associated with the differentiation of PSCs to NPLCs, a process which was indicated to be regulated by miR-124-3p. Overexpression of miR-124-3p resulted in decreased expression levels of FSTL1 during the differentiation of PSCs to NPLCs and dual-luciferase reporter assays identified FSTL1 as a direct target of miR-124-3p. A previous study reported that PSCs can be induced to differentiate to 
NPLCs to repair degenerative intervertebral discs, which can provide unique therapeutic advantages (24). The present study elucidated the function and molecular mechanism underlying the role of miR-124-3p in the differentiation of PSCs to NPLCs. Therefore, it was hypothesized that miR-124-3p may be a target for the induction of PSC differentiation and the subsequent repair of degenerated intervertebral discs.

In conclusion, the present study demonstrated a novel role for miR-124-3p in the differentiation of PSCs to NPLCs, and provided evidence that miR-124-3p negatively regulated its target gene, FSTL1, during PSC differentiation. However, the limitations of the present study lie in the lack of effective in vivo experiments to demonstrate the regulatory effect of miR-124-3p on PSCs. Moreover, whether miR-124-3p can alleviate DDD remains unclear. Therefore, further investigations into the function of miR-24-3p in vivo should be an aim of future studies.

\section{Acknowledgements}

Not applicable.

\section{Funding}

The present study was funded by National Science Foundation of China (grant no. 81101374).

\section{Availability of data and materials}

All data generated and/or analyzed during the present study are included in the published article.

\section{Authors' contributions}

QW designed the current study and acquired funding. JW, $\mathrm{XG}, \mathrm{DF}, \mathrm{DL}$ and TJ performed the experiments, collected the data and wrote the manuscript. QW and JW confirmed the authenticity of all the raw data. All authors have read and approved the final manuscript.

\section{Ethics approval and consent to participate}

The present study was approved by Institutional Animal Care and Use Committee of Wuxi People's Hospital (approval no. WXPH20190103c0600105).

\section{Patient consent for publication}

Not applicable.

\section{Competing interests}

The authors declare that they have no competing interests.

\section{References}

1. Fardon DF, Williams AL, Dohring EJ, Murtagh FR, Gabriel Rothman SL and Sze GK: Lumbar disc nomenclature: Version 2.0: Recommendations of the combined task forces of the North American Spine Society, the American Society of Spine Radiology, and the American Society of Neuroradiology. Spine J 14: 2525-2545, 2014.
2. Hemanta D, Jiang XX, Feng ZZ, Chen ZX and Cao YW: Etiology for Degenerative Disc Disease. Chin Med Sci J 31: 185-191, 2016.

3. Lu H and Peng L: Efficacy and safety of Mobi-C cervical artificial disc versus anterior discectomy and fusion in patients with symptomatic degenerative disc disease: A meta-analysis. Medicine (Baltimore) 96: e8504, 2017.

4. Matta A, Karim MZ, Isenman DE and Erwin WM: Molecular therapy for degenerative disc disease: Clues from secretome analysis of the notochordal cell-rich nucleus pulposus. Sci Rep 7: 45623, 2017.

5. Liang L, Li X, Li D, Jiang W, Wang H, Chen J, Sun Z, Zhang N and Zhu Y: The characteristics of stem cells in human degenerative intervertebral disc. Medicine (Baltimore) 96: e7178, 2017.

6. Guo J, Shao M, Lu F, Jiang J and Xia X: Role of Sirt1 plays in nucleus pulposus cells and intervertebral disc degeneration. Spine (Phila Pa 1976) 42: E757-E766, 2017.

7. Vedicherla S and Buckley CT: Cell-based therapies for intervertebral disc and cartilage regeneration - Current concepts, parallels, and perspectives. J Orthop Res 35: 8-22, 2017.

8. Skovrlj B, Cunn G, Guzman JZ and Qureshi SA: Mesenchymal stem cell technology in the treatment of degenerative disc disease. J Neurosurg Sci 59: 25-35, 2015.

9. Centeno C, Markle J, Dodson E, Stemper I, Williams CJ, Hyzy M, Ichim $\mathrm{T}$ and Freeman M: Treatment of lumbar degenerative disc disease-associated radicular pain with culture-expanded autologous mesenchymal stem cells: A pilot study on safety and efficacy. J Transl Med 15: 197, 2017.

10. Li XC, Tang Y, Wu JH, Yang PS, Wang DL and Ruan DK: Characteristics and potentials of stem cells derived from human degenerated nucleus pulposus: Potential for regeneration of the intervertebral disc. BMC Musculoskelet Disord 18: 242, 2017.

11. Zhang S, Chen A, Hu W, Li M, Liao H, Zhu W, Song D and Guo F: Immunological purification of rat precartilaginous stem cells and construction of the immortalized cell strain. Arch Orthop Trauma Surg 128: 1339-1344, 2008.

12. Li C, Wang $Q$ and Wang JF: Transforming growth factor- $\beta$ (TGF- $\beta$ ) induces the expression of chondrogenesis-related genes through TGF- $\beta$ receptor II (TGFRII)-AKT-mTOR signaling in primary cultured mouse precartilaginous stem cells. Biochem Biophys Res Commun 450: 646-651, 2014.

13. Fan MP, Si M, Li BJ, Hu GH, Hou Y, Yang W, Liu L, Tang B and Nie L: Cell therapy of a knee osteoarthritis rat model using precartilaginous stem cells. Eur Rev Med Pharmacol Sci 22: 2119-2125, 2018

14. Richardson SM,Kalamegam G,Pushparaj PN, Matta C,Memic A, Khademhosseini A, Mobasheri R, Poletti FL, Hoyland JA and Mobasheri A: Mesenchymal stem cells in regenerative medicine: Focus on articular cartilage and intervertebral disc regeneration. Methods 99: 69-80, 2016.

15. Xia K, Gong Z, Zhu J, Yu W, Wang Y, Wang J, Xu A, Zhou X, Tao H, Li F and Liang C: Differentiation of pluripotent stem cells into nucleus pulposus progenitor cells for intervertebral disc regeneration. Curr Stem Cell Res Ther 14: 57-64, 2019.

16. Chen P, Ning L, Qiu P, Mo J, Mei S, Xia C, Zhang J, Lin X and Fan S: Photo-crosslinked gelatin-hyaluronic acid methacrylate hydrogel-committed nucleus pulposus-like differentiation of adipose stromal cells for intervertebral disc repair. J Tissue Eng Regen Med 13: 682-693, 2019.

17. Ma K, Chen S, Li Z, Deng X, Huang D, Xiong L and Shao Z: Mechanisms of endogenous repair failure during intervertebral disc degeneration. Osteoarthritis Cartilage 27: 41-48, 2019.

18. Yang Z, Gao XJ and Zhao X: CDMP1 promotes type II collagen and aggrecan synthesis of nucleus pulposus cell via the mediation of ALK6. Eur Rev Med Pharmacol Sci 24: 10975-10983, 2020.

19. Le Maitre CL, Pockert A, Buttle DJ, Freemont AJ and Hoyland JA: Matrix synthesis and degradation in human intervertebral disc degeneration. Biochem Soc Trans 35: 652-655, 2007.

20. Yang $\mathrm{SH}, \mathrm{Wu} \mathrm{CC}$, Shih TT, Sun $\mathrm{YH}$ and $\mathrm{Lin} \mathrm{FH}$ : In vitro study on interaction between human nucleus pulposus cells and mesenchymal stem cells through paracrine stimulation. Spine (Phila Pa 1976) 33: 1951-1957, 2008.

21. Wang Q, Gu X, Cheng L and Wang J: Differentiation of immortalized human precartilaginous stem cells into nucleus pulposus-like cells. Int J Clin Exp Pathol 8: 2816-2822, 2015.

22. Saliminejad K, Khorram Khorshid HR, Soleymani Fard S and Ghaffari SH: An overview of microRNAs: Biology, functions, therapeutics, and analysis methods. J Cell Physiol 234: 5451-5465, 2019 
23. Wang C, Wang WJ, Yan YG, Xiang YX, Zhang J, Tang ZH and Jiang ZS: MicroRNAs: New players in intervertebral disc degeneration. Clin Chim Acta 450: 333-341, 2015.

24. Wang Q, Gu X and Wang J: Transforming growth factor- $\beta 1$ (TGF- $\beta 1$ ) induces mouse precartilaginous stem cell differentiation through TGFRII-CK1 $1 \varepsilon-\beta$-catenin signalling. Int J Exp Pathol 99: 113-120, 2018.

25. Livak KJ and Schmittgen TD: Analysis of relative gene expression data using real-time quantitative PCR and the 2(-Delta Delta C(T)) method. Methods 25: 402-408, 2001.

26. Zhou X, Tao Y, Chen E, Wang J, Fang W, Zhao T, Liang C, $\mathrm{Li} F$ and Chen Q: Genipin-cross-linked type II collagen scaffold promotes the differentiation of adipose-derived stem cells into nucleus pulposus-like cells. J Biomed Mater Res A 106: $1258-1268,2018$

27. Vishnoi A and Rani S: miRNA biogenesis and regulation of diseases: An overview. Methods Mol Biol 1509: 1-10, 2017.

28. Weber KT, Jacobsen TD, Maidhof R, Virojanapa J, Overby C, Bloom O, Quraishi S, Levine M and Chahine NO: Developments in intervertebral disc disease research: Pathophysiology, mechanobiology, and therapeutics. Curr Rev Musculoskelet Med 8: 18-31, 2015.

29. Kang Q, Xiang Y, Li D, Liang J, Zhang X, Zhou F, Qiao M, Nie Y, He Y, Cheng J, et al: miR-124-3p attenuates hyperphosphorylation of Tau protein-induced apoptosis via caveolin-1-PI3K/Akt/GSK3 $\beta$ pathway in N2a/APP695swe cells. Oncotarget 8: 24314-24326, 2017.

30. Zo RB and Long Z: miR-124-3p suppresses bladder cancer by targeting DNA methyltransferase 3B. J Cell Physiol 234 464-474, 2018

31. Long HD, Ma YS, Yang HQ, Xue SB, Liu JB, Yu F, Lv ZW, Li JY, Xie RT, Chang ZY, et al: Reduced hsa-miR-124-3p levels are associated with the poor survival of patients with hepatocellular carcinoma. Mol Biol Rep 45: 2615-2623, 2018.

32. Luo L, Chi H and Ling J: miR-124-3p suppresses glioma aggressiveness via targeting of Fra-2. Pathol Res Pract 214: 1825-1834, 2018.

33. Zhang Y, Liu HL, An LJ, Li L, Wei M, Ge DJ and Su Z: miR-124-3p attenuates neuropathic pain induced by chronic sciatic nerve injury in rats via targeting EZH2. J Cell Biochem 120: 5747-5755, 2019.
34. Tao Y,Zhou X, Liu D, Li H,Liang C, Li F and Chen Q: Proportion of collagen type II in the extracellular matrix promotes the differentiation of human adipose-derived mesenchymal stem cells into nucleus pulposus cells. Biofactors 42: 212-223, 2016.

35. Lian C, Gao B, Wu Z, Qiu X, Peng Y, Liang A, Xu C, Su P and Huang D: Collagen type II is downregulated in the degenerative nucleus pulposus and contributes to the degeneration and apoptosis of human nucleus pulposus cells. Mol Med Rep 16: 4730-4736, 2017

36. Shibanuma M, Mashimo J, Mita A, Kuroki T and Nose K: Cloning from a mouse osteoblastic cell line of a set of transforming-growth-factor-beta 1-regulated genes, one of which seems to encode a follistatin-related polypeptide. Eur J Biochem 217: 13-19, 1993.

37. Ogura Y, Ouchi N, Ohashi K, Shibata R, Kataoka Y, Kambara T, Kito T, Maruyama S, Yuasa D, Matsuo K, et al: Therapeutic impact of follistatin-like 1 on myocardial ischemic injury in preclinical models. Circulation 126: 1728-1738, 2012.

38. Torres S, Bartolomé RA, Mendes M, Barderas R, Fernandez-Aceñero MJ, Peláez-García A, Peña C, Lopez-Lucendo M, Villar-Vázquez R, de Herreros AG, et al: Proteome profiling of cancer-associated fibroblasts identifies novel proinflammatory signatures and prognostic markers for colorectal cancer. Clin Cancer Res 19: 6006-6019, 2013.

39. Wilson DC, Marinov AD, Blair HC, Bushnell DS, Thompson SD, Chaly Y and Hirsch R: Follistatin-like protein 1 is a mesenchyme-derived inflammatory protein and may represent a biomarker for systemic-onset juvenile rheumatoid arthritis. Arthritis Rheumatism 62: 2510-2516, 2010.

40. Liu Y, Wei J, Zhao Y, Zhang Y, Han Y, Chen B, Cheng K, Jia J, Nie L and Cheng L: Follistatin-like protein 1 promotes inflammatory reactions in nucleus pulposus cells by interacting with the MAPK and $\mathrm{NF \kappa B}$ signaling pathways.Oncotarget 8: 43023-43034, 2017.

This work is licensed under a Creative Commons Attribution-NonCommercial-NoDerivatives 4.0 International (CC BY-NC-ND 4.0) License. 